\title{
More Use of Peritoneal Dialysis Gives Significant Savings: A Systematic Review and Health Economic Decision Model
}

\author{
Eva Pikea, Vida Hamidia, Tove Ringerike ${ }^{\mathrm{a}}$, Torbjorn Wisloffa, ${ }^{\mathrm{b}}$, \\ Marianne Klempa ${ }^{\mathrm{a}, \mathrm{b}, \mathrm{c}}$
}

\begin{abstract}
Background: Patients with end-stage renal disease (ESRD) are in need of renal replacement therapy as dialysis and/or transplantation. The prevalence of ESRD and, thus, the need for dialysis are constantly growing. The dialysis modalities are either peritoneal performed at home or hemodialysis (HD) performed in-center (hospital or satellite) or home. We examined effectiveness and cost-effectiveness of HD performed at different locations (hospital, satellite, and home) and peritoneal dialysis (PD) at home in the Norwegian setting.
\end{abstract}

Methods: We conducted a systematic review for patients above 18 years with end-stage renal failure requiring dialysis in several databases and performed several meta-analyses of existing literature. Mortality and major complications that required were our main clinical outcomes. The quality of the evidence for each outcome was evaluated using GRADE. Cost-effectiveness was assessed by developing a probabilistic Markov model. The analysis was carried out from a societal perspective, and effects were expressed in quality-adjusted life-years. Uncertainties in the base-case parameter values were explored with a probabilistic sensitivity analysis. Scenario analyses were conducted by increasing the proportion of patients receiving PD with a corresponding reduction in HD patients in-center both for Norway and Europian Union. We assumed an annual growth rate of $4 \%$ in the number of dialysis patients, and a relative distribution between PD and HD in-center of $30 \%$ and $70 \%$, respectively.

Results: From a societal perspective and over a 5-year time horizon, PD was the most cost-effective dialysis alternative. We found no significant difference in mortality between peritoneal and HD modalities. Our scenario analyses showed that a shift toward more patients on PD (as a first choice) with a corresponding reduction in HD incenter gave a saving over a 5-year period of 32 and 10,623 million EURO, respectively, for Norway and the European Union.

Conclusions: PD was the most cost-effective dialysis alternative and

Manuscript accepted for publication November 22, 2016

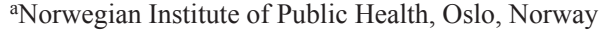

${ }^{b}$ Department of Pharmacology, University of Oslo, Norway

'Corresponding Author: Marianne Klemp, Norwegian Institute of Public

Health, PB 4404, Nydalen, 0403 Oslo, Norway.

Email: marianne.klemp@medisin.uio.no

doi: https://doi.org/10.14740/jocmr2817w was comparable with HD regarding efficacy outcomes. There are significant saving potentials if more end-stage renal patients are started on PD instead of HD.

Keywords: Dialysis; Peritoneal; Hemodialysis; Health economics

\section{Introduction}

Patients with end-stage renal disease (ESRD) have a need for renal replacement therapy (RRT) as either dialysis and/or transplantation. ESRD is the last stage of chronic kidney disease (CKD) [1]. The prevalence of CKD is estimated to be $8-16 \%$ worldwide [2]. The number of patients with CKD and ESRD is rising worldwide with the growth rate higher than the growth rate for general population $[2,3]$. The reason for this rise is that one expects a rise in number of patients with high blood pressure and diabetes [2], further the expected demographic development with increased numbers of elderly people also contributes, since the prevalence of CKD is higher in older people [4]. Factors as higher life-expectancy of treated ESRD patients and increasing access of a generally younger patient population to treatment in countries in which access had previously been limited also contribute [5].

The number of patients with ESRD receiving dialysis globally was estimated to be 2,358,000 at the end of 2012 [6]. The annual growth rate of dialysis patients was between $2 \%$ and $7 \%$, respectively in the European Union (EU) and globally at the end of $2012[5,6]$. The number of dialysis patients in Norway has increased from 241 in 1990 to 1,240 in 2012 [7].

RRT consists of either dialysis and/or transplantation. Patients are normally starting on dialysis while waiting for a renal graft, and not all patients receiving RRT will be transplanted due to shortage of renal grafts. Generally, there are two different types of dialysis: hemodialysis (HD) and peritoneal dialysis (PD). HD can be performed in hospitals, different medical institutions or at home. However, HD is most often performed in dialysis centers (hospitals including self-care and satellite units) [6]. PD is carried out at home by the patients, often without assistance from healthcare workers.

Globally, at the end of 2012, around eight times more patients received $\mathrm{HD}$ as compared to $\mathrm{PD}$, i.e., $\mathrm{PD}$ patients represented only $11 \%$ of the dialysis population [6]. In Norway, HD performed in hospitals (satellites included) is the most fre- 
quently used modality (84.2\%), whereas PD at home makes up for $15.8 \%$ [7].

With the expected increase in demand for dialysis, it is essential to assess which dialysis modality is the most advantageous compared to the others. Our aim was therefore to compare both effectiveness and cost-effectiveness of HD at different locations and PD in the Norwegian setting and to calculate possible savings from starting more ESRD patients on PD.

\section{Materials and Methods}

\section{Clinical efficacy}

We conducted a systematic review (SR) and several metaanalyses of existing literature, following the methods recommended in the PRISMA statement for reporting systematic reviews and meta-analyses of studies that evaluate healthcare interventions [8]. The protocol for the SR can be seen in Supplementary 1 (www.jocmr.org).

\section{Eligibility criteria}

We included SRs, health technology assessments (HTAs), randomized controlled trials (RCTs) and observation studies with control group that assessed mortality and complications in patients above 18 years with end-stage renal failure requiring dialysis.

\section{Interventions/controls}

All the different dialysis modalities were compared with each other: 1) HD carried out in hospital (HD hospital), 2) self-care HD in hospital (HD self-care), 3) HD in satellite unit (nursing home and local medical center) (HD satellite), 4) HD home and 5) PD at home.

\section{Endpoints}

Our clinical endpoints were mortality and major complications that required hospitalization.

\section{Literature search}

Our SR was based upon a systematic literature search performed in May 2012, and updated in August 2013 and October 2014. We updated again the search in February 2016, and the results of that were only included in the discussion. The time period for the search was limited from 1995 to the date for the search. The reason for choosing 1995 as the starting point was that erythropoietin was introduced about that time. The complete search strategies are presented in Supplementary 2 (www.jocmr.org). We searched the following databases: The Cochrane Library, Centre for Reviews and Dissemination,
Ovid MEDLINE(R), and EMBASE (Ovid). We checked the reference lists in SRs that we reviewed in full text, and performed manual searches in the following websites: INAHTA (International Network of Agencies for Health Technology Assessment), Clinical Evidence, ISI Web of Knowledge, NHS Evidence, AHRQ (Agency for Healthcare Research and Quality's), SBU (Swedish Council on Health Technology Assessment), Dacehta, Finohta/THL (National Institute for Health and Welfare), CADTH (Canadian Agency for Drugs and Technologies in Health), AHTA (Adelaide Health Technology Assessment), NIHR (National Institute for Health Research), and NICE (National Institute for Health and Care Excellence).

\section{Selection of articles}

Two reviewers independently inspected all citations to identify potentially relevant articles based on title and/or abstract. Full text publications were obtained for articles appearing to meet our inclusion criteria. Two persons independently assessed whether the article was relevant or not according to our list of inclusion criteria. Disagreements were resolved by discussion or by consulting a third reviewer.

To be certain that the estimates of efficacy or safety reflected the delivered treatments, and not different prognostic features of the patients, we examined the baseline data of patients included in the studies. If differences in comorbidity between groups were reported or detected by our own analysis, or if no description of the patient's comorbidity was reported, the study was excluded. However, if the article provided analyses that adjusted for this difference, the study was included in our assessments.

\section{Data extraction and analyses}

One reviewer extracted data from the included articles and another reviewer checked these results for accuracy. When data were presented in several ways, we chose to report data in our preferred order: hazard ratio (HR), relative risk (RR) and odds ratio (OR) with 95\% confidence intervals (CIs). We reported adjusted data if adjustments seemed reasonable. When possible, we performed meta-analyses using RevMan 5.2 software using a random effects model. In cases where both events and patients at risk were available from the publications for all studies for a specific outcome, RRs were calculated using the Mantel-Haenszel approach in Review Manager. When the data regarding the same outcome were reported in different ways in the included publications, we re-calculated to log risk ratios and standard error, and the common RR was calculated using inverse variance in Review Manager. Supplementary 3 (www. jocmr.org) gives a description of transformation of the results to $\log \mathrm{RR}$ and standard error.

\section{The quality of the evidence}

The quality of the included studies was assessed using the 


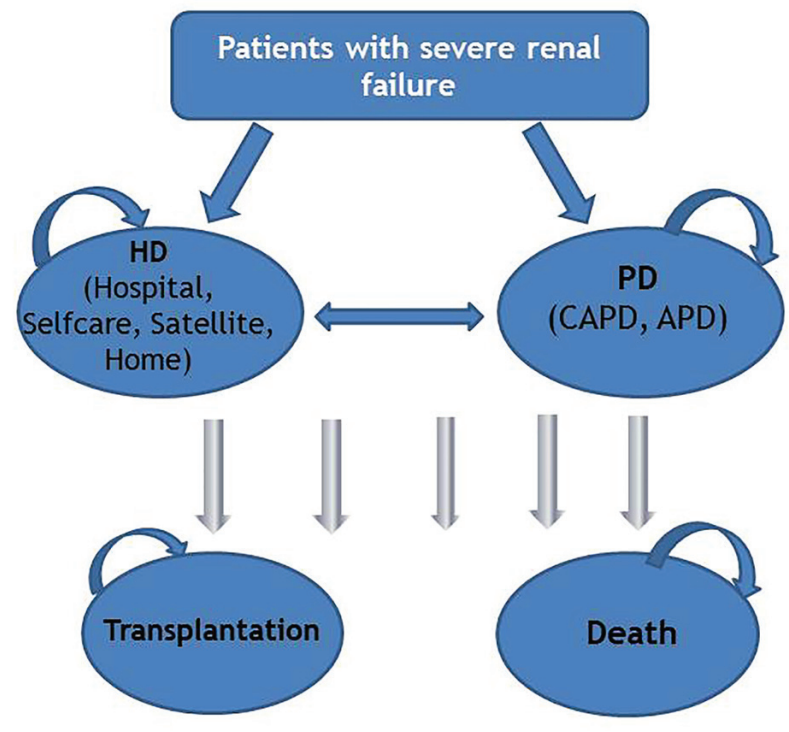

Figure 1. Model structure. HD: hemodialysis; PD: peritoneal dialysis; CAPD: continuous ambulatory peritoneal dialysis; APD: automated peritoneal dialysis.

Cochrane Collaboration's tool for risk of bias assessment [9]. The quality of the evidence for each outcome was assessed by using GRADE (Grading of Recommendations, Assessment, Development and Evaluation) [10]. In the GRADE system, outcome documentation from RCTs starts at high quality and outcome documentation from observational studies starts at low quality. The method involves an evaluation of study type, study quality/risk of bias, consistency between trials, directness (how similar the population, intervention, and outcomes are between the trials and the objectives of this SR) and precision of the estimates. Finally, the overall quality will be categorized as high, moderate, low or very low. All assessments were performed and agreed upon by at least two reviewers.

\section{Cost-effectiveness analysis}

\section{Model structure}

In order to assess the cost-effectiveness of different dialysis modalities, a decision analytic model was developed in TreeAge pro ${ }^{\circledR}$ 2012. The model is of the Markov type, in which a cohort of patients is followed over a given period of time. A Markov model was considered appropriate as ESRD is a chronic condition requiring continuous treatment and interaction between patients is not likely to have influence on neither prognosis nor costs.

The model assumes that patients with severe renal failure start in one of the dialysis modalities: HD hospital, HD selfcare, HD satellite, HD home and PD. Three states, HD home, HD satellite, and PD, include a stabilization and training period in the hospital. Transplantation is included in the model to represent all of the possible modalities affording RRT for ESRD patients, but only as absorbing state. Once an individual makes a transition into the absorbing state, no further incurred costs are included in the analysis. Based on the Norwegian renal registry annual reports, median time from start of RRT until death is $33-42$ months over the last few years [7]. A 5-year time horizon was therefore used to assess the clinical and economic outcomes associated with each treatment strategy. The cycle length of the model was 1 year, meaning that any transitions between different states could happen only once per year. Patients could be in only one of the pre-defined states at any time. Upon completion of each cycle patients could, depending on transition probabilities, transfer to another state or remain in the same state until death or the end of the simulation. In addition, patients could experience complication events during each health state. Each state and event is associated with specific outcomes and costs. The model structure is presented in Figure 1.

The analysis was carried out from a societal perspective. Both costs and effects were discounted at a rate of $4 \%$ per annum. The results of the economic evaluations were expressed as mean incremental cost-effectiveness ratio (ICER) and mean incremental net health benefit (INHB).

\section{Model parameters}

\section{1) Probabilities}

The probability of transferring to another state or remaining in the same state was estimated based on Norwegian epidemiological data and clinical efficacy estimates. The transition probabilities, i.e. the probability of starting in one modality and switching to another, are presented in Table 1 [11-21]. All of the base-line probabilities were based on data from the Norwegian renal registry over a 5-year time horizon [11], i.e. a cohort of patients who started dialysis in 2007 and were followed for 5 years. The registry data were divided into HD, PD and transplant patients. Yearly mortality from the registry was combined for all different dialysis modalities. In the model, this combined mortality rate was multiplied by the estimate of RRs of death from our SR.

\section{2) Clinical efficacy parameters}

Clinical efficacy data for the model were derived from our SR of mortality for the comparisons PD home versus HD hospital and HD home versus HD satellite. The RRs were added to the model as log-normal distributions (Supplementary 4, www.jocmr.org). Our SR showed that there is lack of evidence comparing all different HD modalities. As we needed a common comparator in the model, it was assumed that there is no difference in mortality between HD hospital and HD satellite.

\section{3) Costs}

An annual cost per patient associated with the treatment modalities was calculated for each health state in the model. We 


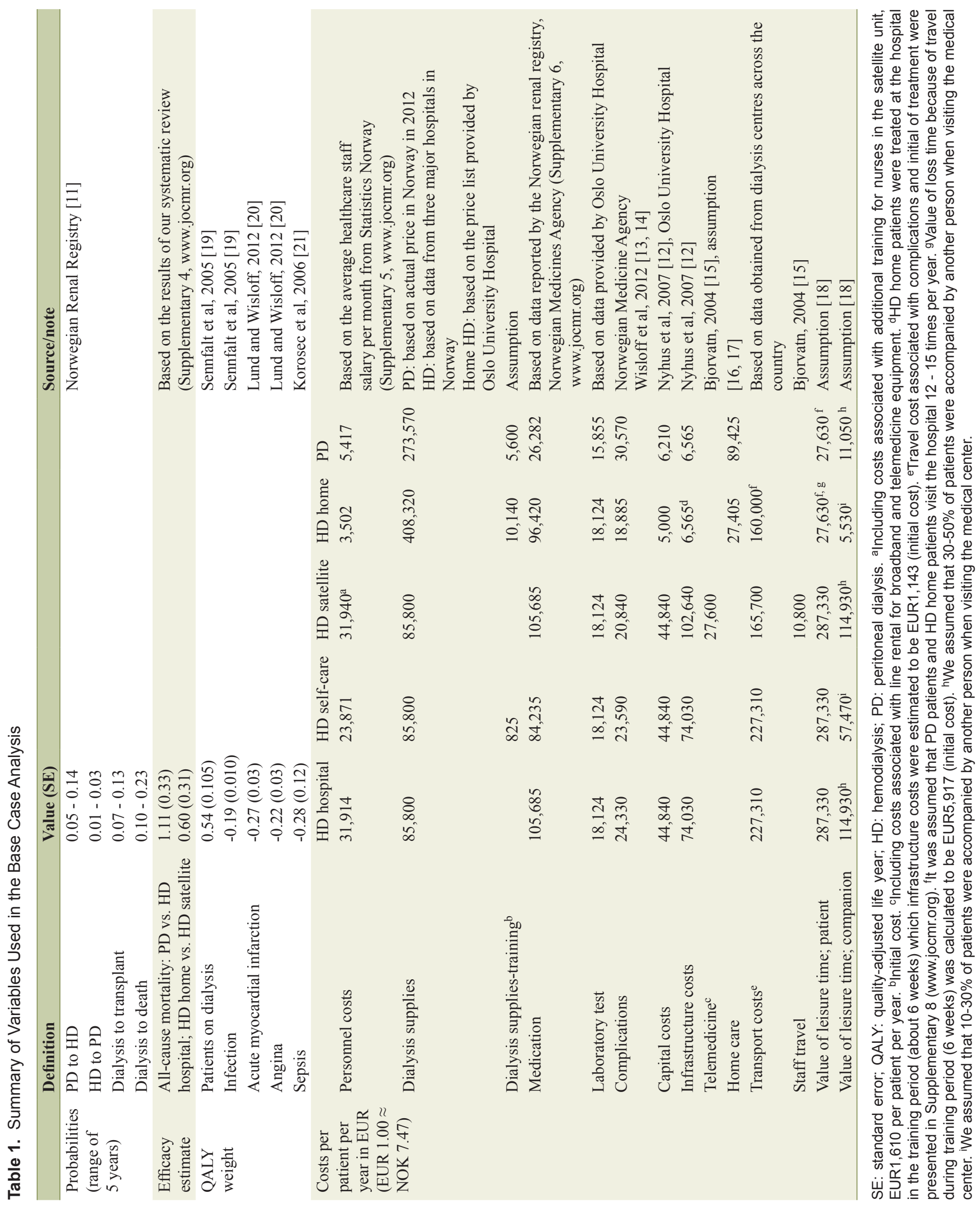


present the results from a societal perspective, including direct health care costs as well as indirect costs related to dialysis treatment. We have attempted to identify and quantify the differences between the treatment options and less emphasis on common elements.

Direct costs for dialysis care include costs associated with personnel (physicians, nurses and other involved personnel), medicines, supplies, laboratory tests, complications of the dialysis, training, as well as other costs borne by hospitals (e.g. costs associated with telemedicine communication for satellite units, capital and infrastructure costs) and transport cost. All costs were measured in 2012 Norwegian kroner (NOK) and translated into EUROs (EUR1.00 $\approx$ NOK7.47) and are presented in Table 1.

The costs of personnel involved in dialysis treatment were calculated based on estimates of staff time per dialysis session for different treatment modalities (Supplementary 5, www.jocmr.org). For PD and HD home, the calculation was based on the time required for initiation of treatment, training of patient for the procedure, consultation and outpatient visits. The mean cost per hour was estimated based on the average healthcare staff salary per month from Statistics Norway [22].

Costs of dialysis supplies for HD hospital were obtained from a Norwegian study based on data from three major hospitals in Norway [12]. The costs were updated to 2012 costs. Lacking specific data, we assumed that the cost of consumable supplies for satellite and HD self-care was the same as the related costs for HD hospital. It is possible, however, that the cost of supplies varies across settings. Costs of dialysis supplies for HD home were based on the price list provided by Oslo University Hospital.

The consumable supplies costs for PD were the actual price in Norway in 2012. The consumable costs for automated peritoneal dialysis (APD) are more expensive than for continuous ambulatory peritoneal dialysis (CAPD). We estimated the costs for PD based on the assumption that $60 \%$ of PD patients use CAPD [11].

Drug costs were calculated based on maximum pharmacy retail prices from the Norwegian Medicines Agency [13]. Drug doses were estimated based on treatment guidelines and expert opinion. In our analysis, we have only included costs associated with those medications for which considerable differences in use were reported for HD versus PD patients (based on data obtained from the Norwegian Renal Registry) (Supplementary 6, www.jocmr.org). In addition, we included the cost associated with anticoagulation during HD therapy, and treatment of iron deficiency anaemia (as different drugs and administration methods used for treatment of HD and PD patients) in our analysis. We assumed identical drug costs for all HD modalities.

Annual laboratory test costs were calculated separately for HD and PD patients based on the standard blood tests for dialysis patients and the price lists provided by Oslo University Hospital.

We have included two types of major complications that require special measures in our model: infections and cardiovascular event. For PD, we included peritonitis and sepsis; while for HD, we included infections (access-related infections and sepsis) and cardiovascular events (acute myocardial infarction (AMI) and angina) in the analysis. Probabilities of the occurrence of these events were estimated based on data from the Norwegian Renal Registry [11] (Supplementary 7, www.jocmr.org). Probabilities of access-related hospitalization and cardiovascular events were adjusted according to different dialysis modalities by multiplying the probabilities taken from Norwegian renal registry by the relative risks of these events from our SR (Supplementary 8, www.jocmr.org). The costs of treating infections associated with dialysis treatment have been calculated based on the treatment recommended in the National guidelines for antibiotic use in hospitals [23]. We assumed patients received inpatient care for an average of 7 - 10 days per infection (expert opinion). The cost of cardiovascular events was calculated based on a previous Norwegian economic evaluation [14]. For cardiovascular events, we included the costs of two most common interventions related to coronary artery surgery (AMI and angina) in our analysis [24, 25]. The costs of treating septicemia were also estimated based on a Norwegian study [26].

Costs related to equipment (investment commodities) were estimated based on data from three major hospitals in Norway [12]. Costs associated with water system, computers, ECG machine, infusion pump, blood pressure measure, warming plate and the other equipment (e.g. TV, beds furniture, weights ivrack, etc.) were included in the analysis. An equivalent annual cost was calculated for equipment items over relevant lifespans for the items, using a $4 \%$ discount rate. The lifetimes for the water system and the other equipment were set at 10 and 5 years, respectively. Capital costs associated with home HD treatment were estimated for using the new type of machine, which requires less installation and reconstruction than standard HD machines.

Infrastructure costs were calculated based on a Norwegian study [12]. The infrastructure costs for HD and PD were estimated to be approximately EUR880 and EUR74 per patient per month, respectively. Due to uncertainly around the estimations, we varied overhead costs in the probabilistic sensitivity analysis. We used a gamma distribution with a standard error of 780.66 for HD and 75.06 for PD. For HD home, we assumed the same overhead cost as PD (15 visits to the hospital per year). Further, HD home patients were treated at the hospital in the training period (about 6 weeks). Overhead costs for HD satellite were estimated based on data reported by Bjorvatn [15]. The costs were updated to 2012 prices (approximately EUR1,145 per patient per month with a standard error of 1,309).

We included in our analysis the cost related to installation of telemedicine equipment for satellite units based on the costs reported by Bjorvatn [15]. We calculated the cost based on the assumption of a 5-year lifetime for the telemedicine equipment and a discount rate of $4 \%$ per year. These costs were updated to 2012 costs (approximately EUR1,473 - EUR1,740 per patient, annually). Line rental for broadband was assumed to be EUR1,606 - EUR2,410 per patient, annually.

The average distance traveled to the unit (hospital or satellite unit) was calculated based on data obtained from dialysis centers across the country. The average travel cost per Scandinavian mile $(10 \mathrm{~km})$ was estimated to be EUR44. It was assumed that the average number of hospital visits/treatments 
per year is 156 for HD hospital and HD satellite patients, and 15 for PD and HD home patients (including visiting a nephrologist and initial training). Moreover, we have estimated travel cost associated with initiation of treatment (PD, HD home and HD satellite) and treatment of complications (Supplementary 9, www.jocmr.org). For HD satellite, we have also included the costs associated with staff travel (for nurses and physicians).

A Norwegian study has shown that about $30-40 \%$ of PD patients required home care assistance related to the treatment (e.g. fluid exchange) [16]. We also assumed that $10-20 \%$ of HD home patients might require home care help. The estimated cost of one nurse visit (assumed one hour) was EUR94 [17].

We conducted our analyses from a "limited" societal perspective, which include costs that may be borne by patients and their families (i.e., value of lost time due to travel). Costs linked to productivity loss were not included in our analysis, as elderly patients account for an increasing fraction of patients on RRT [18]. The reported average age of the patients undergoing dialysis in Norway during the last 5 years is approximately 62 years [11]. Moreover, the result of a cohort study among Norwegian dialysis patients indicated that none of the patients was working, two-thirds of patients were retired and the remaining one-third was receiving a disability pension benefit [15]. Therefore, only the value of leisure time for patients and value of lost time for any accompanying people were included in the analysis.

Value of leisure time lost to travel was estimated by multiplying lost leisure time $(4-8 \mathrm{~h})$ by the annual number of treatments and the national average hourly wage rate [18]. For patients undergoing dialysis in the hospital (both HD hospital and HD self-care) and satellite unit, we assumed one workingday as lost leisure time per treatment. For patients undergoing treatment at home (HD home and PD), the estimated average number of hospital visiting days per year was 15 and the leisure time loss was assumed to be one working-day at each visit.

We assumedthat $10-50 \%$ of patients were accompanied by another person when visiting the medical centre (hospital or satellite unit). We also assumed that companions did not participate in the labor market, therefore only the value of leisure time for companions has been included in the analysis.

In our analysis from the societal perspective, we have deducted value-added tax and other transfer payments to the government from the included direct costs.

\section{4) Health-related quality of life}

Previous published studies showed no significant quality of life differences based on dialysis type or treatment setting. To avoid bias in favor of one of the modalities, we applied the standard error-adjusted mean of the HD and PD EQ-5D utilities reported in a Swedish matched-case study [19] as the single QALY weight of $0.54(0.34-0.75)$ for all types of dialysis in the model (Table 1). This estimate was compatible with the results reported by Liem and co-authors [27] of a meta-analysis of quality of life results measured using the EQ-5D instrument. Because EQ-5D is the most used instrument to measure healthrelated quality of life in health economic evaluations [28], we used data based on this instrument seeking to achieve better concordance between economic evaluations. We assumed that all patients were in the same underlying health state when entering the model.

Because of the lack of quality of life data for dialysis patients experiencing complications associated with cardiovascular events, utility losses associated with these events were estimated using the best available data, a Norwegian study of stroke patients from 2011 [20].

\section{Sensitivity analysis}

To explore the consequences of uncertainties in the basecase parameter values, we performed a probabilistic sensitivity analysis in which all input parameters were randomly drawn from probability distributions and the model was run 1,000 times. For probabilities and utility estimates, the betadistributions were constructed on plausible ranges for parameters. For cost, we modeled gamma-distributions where limits were within a $30 \%$ of the base-case value. Efficacy and safety parameters were assumed to have a log-normal distribution where mean and CIs from our own meta-analysis were used to estimate the parameters.

\section{Scenario analyses}

We performed scenario analyses by increasing the proportions of patients receiving $\mathrm{PD}$ with a corresponding reduction in $\mathrm{HD}$ in-center. The scenario analyses were based on the assumption that more patients received $\mathrm{PD}$ as a first choice relative to HD dialysis. The analyses were based upon the total cost given in the base-case cost-effectiveness analyses from the societal perspective. We conducted the scenario analyses both for Norway and for EU. In the scenario analyses for EU, we used the Norwegian cost data as we could not find reliable cost estimates for EU.

\section{Results}

\section{Clinical efficacy}

Our search for literature up to August 2013 identified 109 SRs/ HTAs, 4,346 controlled studies and additionally two controlled studies through manual searches. Finally, 13 publications were included (Fig. 2). Of the 13 publications included, one was an RCT [29] and 12 were publications with data from 13 observational studies [30-41]. Six of the studies were performed in Europe, four in the USA, two in Asia, and one in Canada. More details of the studies are given in Supplementary 10 (www. jocmr.org). A full list of the excluded studies and the reason for the exclusion is presented in Supplementary 11 (www.jocmr. org). An updated search from October 2014 resulted in no further studies that fulfilled our inclusion criteria.

We assessed baseline comorbidity between the patient groups in the included studies and found no significant differences in baseline comorbidity (Supplementary 10, www. 


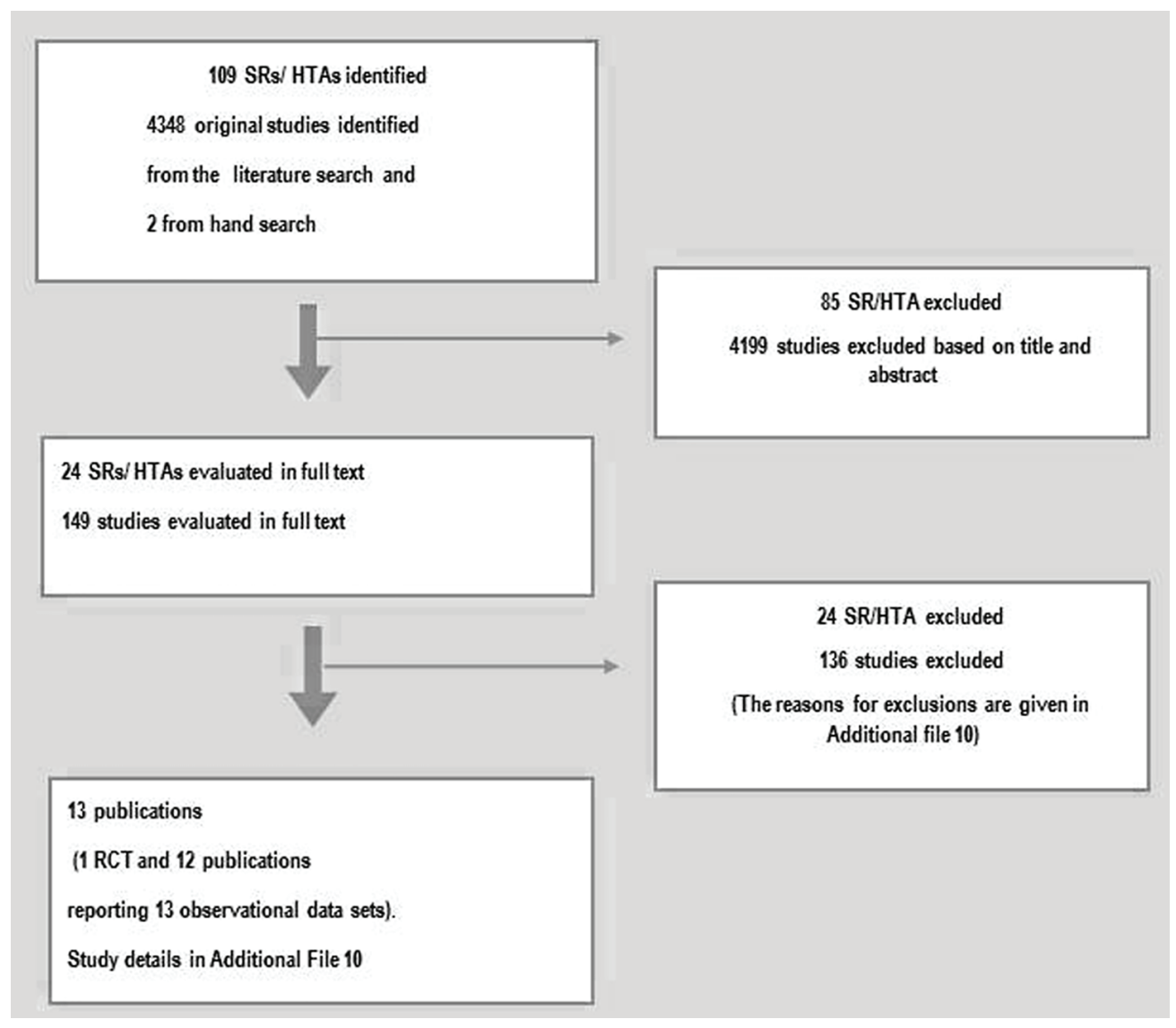

Figure 2. Process of study selection.

jocmr.org). We assessed the risk of bias for the included RCT to be high [29]. All the observational studies were assessed to have high risk of bias, mainly due to lack of randomization (Supplementary 12, www.jocmr.org). The quality of the evidence for each outcome using GRADE ranged from low to very low. Of 10 possible comparisons, only five had data usable for our analyses. Supplementary 13 (www.jocmr.org) shows the possible comparisons and outcomes reported for the specific comparisons. We found no significant differences in mortality between the dialysis modalities for PD versus HD hospital and HD home versus HD satellite (Supplementary 14A and F, www.jocmr.org). For the comparison PD versus HD satellite, there was a significant difference in mortality in favor of PD. However, this comparison was based on only one observational study of very low quality (Supplementary 14D, www.jocmr.org). In our economic modeling analyses for mortality, we used the comparisons PD versus HD hospital and HD home versus HD satellite. For those, the RRs for mortality were $1.11(0.59-2.10)$ and $0.60(0.33-1.10)$, respectively. The complications used in our economic modeling analyses were hospitalization due to access and cardiac or vascular hospitalization. The RRs for access-related hospitalization were 0.88 (0.64 - 1.22) and 1.08 (0.62 - 1.88), respectively, for HD hospital versus HD satellite and for HD home versus HD satellite. The RRs for cardiac or vascular hospitalization were 0.53 $(0.28-1.01), 0.03(0-0.54)$ and $1.45(0.49-4.36)$, respective- ly, for HD satellite versus HD hospital, PD versus HD hospital and HD home versus PD. For more information, see Supplementary 9 and 14B, C, G and $\mathrm{H}$ (www.jocmr.org). All major complications reported were evaluated to very low quality, so the results were uncertain. The very low quality was mostly due to wide CIs, only one study per outcome and few events. For more details about the clinical results, see Supplementary 14 (www.jocmr.org).

\section{Cost-effectiveness}

Table 2 illustrates the results of the base-case analysis. From a societal perspective and over a 5-year time horizon, PD was the most cost-effective dialysis alternative compared to all the HD types. HD home dominated all other HD modalities (i.e. HD hospital, HD self-care and HD satellite). HD home was slightly more effective, but at the same time more costly relative to $\mathrm{PD}$. Therefore, the incremental cost per effect was clearly above the threshold for cost-effectiveness considered cost-effective in the Norwegian setting.

\section{Sensitivity analyses}

The probability of the alternatives being cost-effective subject 
to different levels of willingness to pay (WTP) is shown in cost-effectiveness acceptability curves (Fig. 3a for the comparison of PD and HD (home and in-center) and for the internal comparison within the HD group as shown in Fig. 3b).

The result revealed that among all dialysis modalities and from a societal perspective, PD was likely to be the most costeffective strategy for all values of WTP (Fig. 3a).

Among the HD modalities, HD home was likely to be the most cost-effective startegy (Fig. 3b).

\section{Scenario analyses with starting more patients on PD}

We have performed scenario analyses examining possible savings when more patients start with PD by increasing the proportion of dialysis patients receiving PD (as a first choice) with a corresponding reduction in in-center HD. In Norway, 1,240 patients received dialysis in 2012, and around $15 \%$ of them were treated with PD. In our scenario analyses, we assumed an increase in the annual growth rate of the number of dialysis patients from $2 \%$ to $4 \%$ and a relative distribution between PD and HD in-center of $30 \%$ and $70 \%$, respectively. This gave a possible saving of around 32 million EURO over a 5-year period. These assumptions gave an increase from 1,240 (in 2012) to 1,510 dialysis patients in a 5-year perspective in Norway.

In EU, around 300,000 patients received dialysis in 2012. This is calculated based upon the patient numbers in 2004 [5] and the annual growth rate of $2 \%$ [6]. About $10 \%$ of the dialysis patients in EU are treated with PD [42]. In our scenario analysis, we assumed an annual growth rate of $4 \%$ in the total number of dialysis patients and increased the proportion of PD patients to $30 \%$. This gave a possible cost-saving of around 10,623 million EUROs over a 5-year period. This resulted in an increase from approximately 300,000 (in 2012) to 365,000 dialysis patients in a 5-year perspective. For EU, we also performed a scenario where we increased the proportion of PD patients from $10 \%$ to $20 \%$ (conservative scenario). The scenario analyses from a societal perspective are shown in Table 3.

\section{Discussion}

We have compared efficacy, safety and cost-effectiveness of the different dialysis modalities: 1) HD carried out in hospital, 2) self-care HD in hospital, 3) HD in satellite units (nursing home and local medical center), 4) HD at home and 5) PD at home for patients above 18 years with end-stage renal failure requiring dialysis in Norway.

Overall, there was no clear best choice between dialysis modalities regarding relative efficacy measured as mortality or complications. However, the results showed a trend towards a lower mortality in HD patients (no statistically significant). The results from our health economics evaluations indicated that the four HD dialysis options all were slightly more effective than PD but at the same time more costly and therefore not considered cost-effective relative to PD in the Norwegian setting.

Generally there seems to have been an increase in dialysis

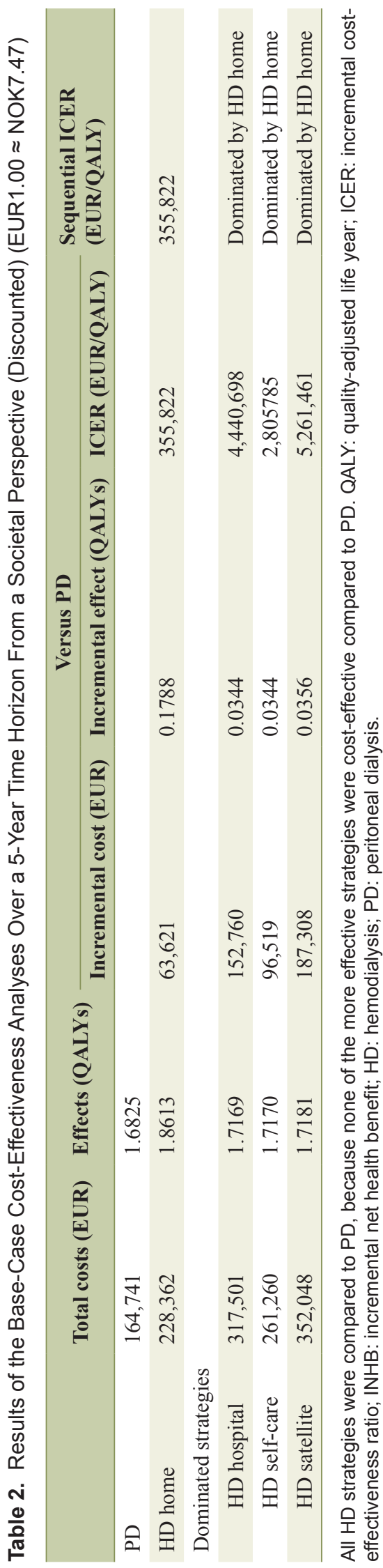



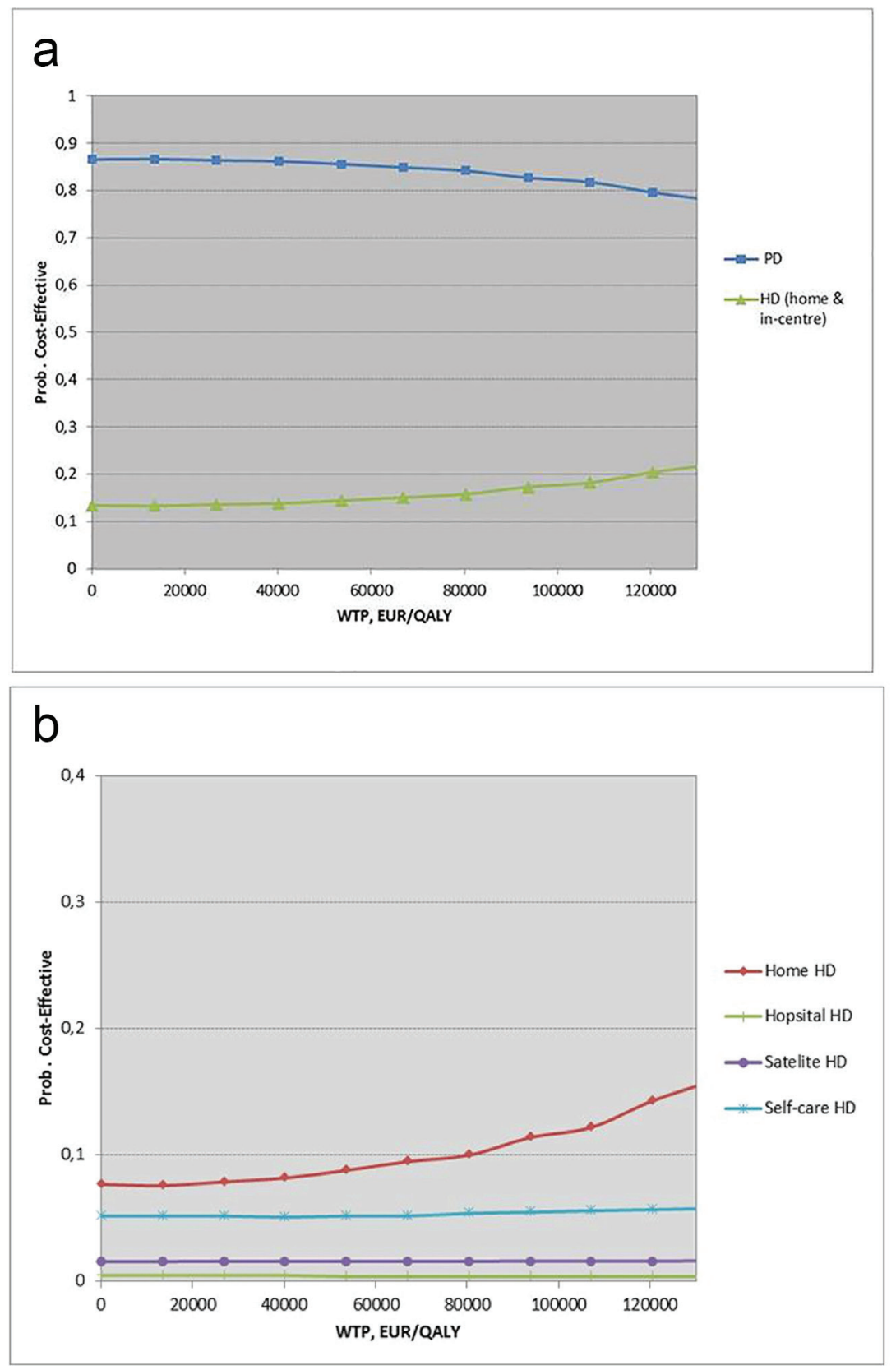

Figure 3. (a) Cost-effectiveness acceptability curve (societal perspective). (b) Cost-effectiveness acceptability curve (societal perspective) for the internal comparison within the HD group. HD: hemodialysis; PD: peritoneal dialysis; WTP: willingness to pay.

survival during the past 20 years [43]. The survival for PD since 2000 has been better than HD, overall and for subgroups [43].

Our results for clinical endpoints are in accordance with published studies showing mortality to be equal or lower for $\mathrm{PD}$, used as initial therapy, compared to HD in hospital for the first 1 - 3 years [44-47]. From 3 years and more, HD reported a survival advantage over PD [44, 45, 47, 48]. In our SR, we included studies with a follow-up time up to 5 years. The patients in the included studies in our SR did not differ significantly in comorbidity, including diabetes, and were similar in age, at baseline. It has been shown that for patients above 65 with diabetes or cardiovascular disease, there may be a survival advantage in the HD group [47]. However, an SR from 2014 [49] showed that there is inconsistent evidence to which modality is the best initial treatment for diabetics patients with ESRD.

Several published studies compare the cost-effectiveness of $\mathrm{PD}$ with $\mathrm{HD}$. As we have shown in our analysis, home dialysis (PD) and HD home are cost-effective or cost-saving compared with in-center HD in most countries [50-54]. However, generally PD is a significantly less expensive dialysis modality 
Table 3. Cost-Saving With an Increased Proportion of Patients Starting on PD at the Expense of In-Center HD Over 5-Year Time Horizon From a Societal Perspective (EUR1.00 NOK7.47)

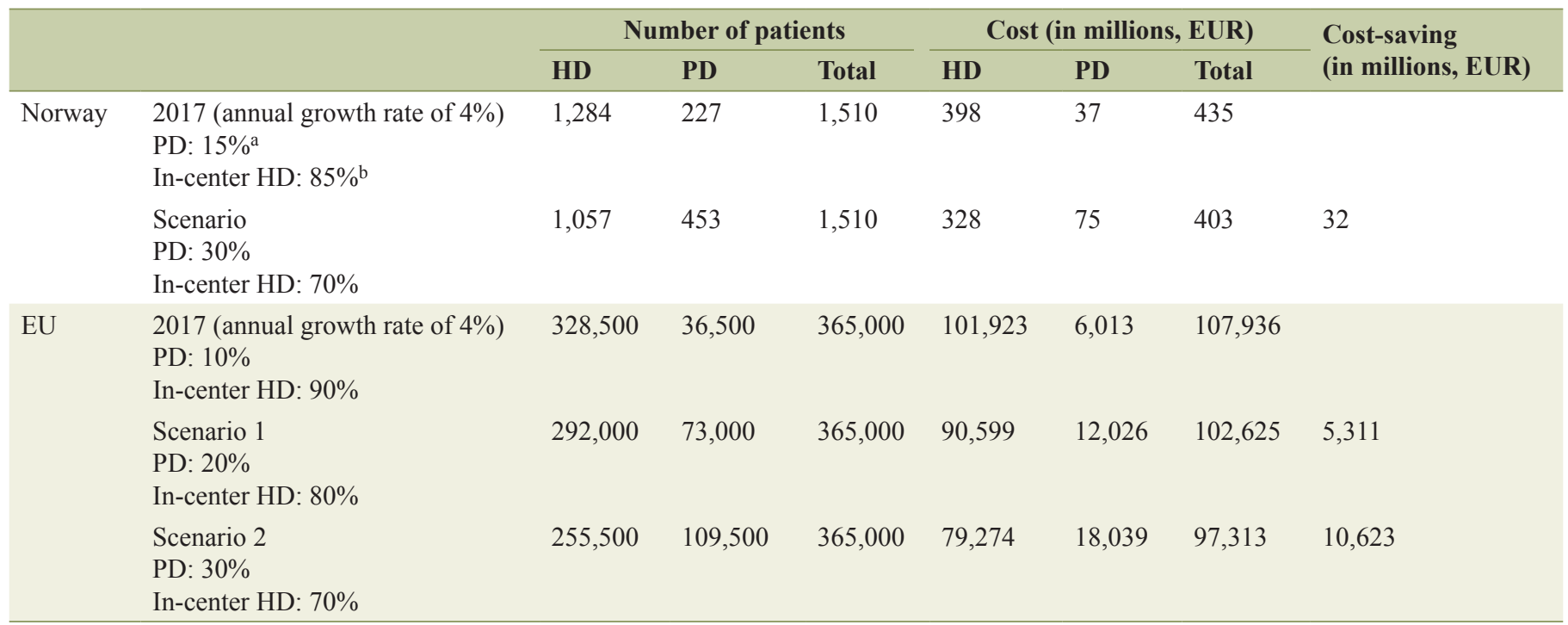

HD: hemodialysis; PD: peritoneal dialysis; EU: The European Union. ${ }^{a}$ In Norway PD in 2012 was actual 15.8\%. ${ }^{b}$ In-center HD includes HD in hospital and satellites.

than HD [52]. This result is largely driven by lower transportation costs to the center, staff costs and value of leisure time lost. Therefore, extending the proportion of patients starting on PD may result in cost-savings for society.

In summary, our own results and results from others have shown PD to be the most cost-effective dialysis modality, which gave lower mortality for the first 3 - 5 years when given as initial dialysis. Based upon this evidence, we showed that there is a substantial potential for cost-saving with a shift towards starting more patients on PD with a corresponding reduction in in-center HDA similar finding has been shown by a study performed in the United Kingdom [55]. Treharne and coautors showed that increasing PD use among incident dialysis patients would be cost-effective and associated with reducing costs [55].

At the end of year 2012, still most dialysis patients globally received $\mathrm{HD}$ in dialysis centers, and only $11 \%$ dialysis patients were undergoing PD $[6,42]$. Several countries had however a proportion of PD patients above $20 \%$ such as in New Zealand, Australia and several countries in the northern Europe [42]. If our scenario with $30 \%$ of the dialysis patients starting on PD should be realistic in most countries, nephrologists would have to make their therapeutic choices based upon evidence from research studies showing that PD is at least equally effective as HD and more cost-effective. However, patient's clinical status and preferences also need to be considered on an individual basis.

\section{Strengths and weaknesses of this study}

To the best of our knowledge, this is the first study that evaluates the relative costs and effects of all dialysis modalities based on an SR.
Lack of data comparing different HD modalities (with regard to treatment location) was the most important limitation of this study. For comparison of efficacy, of 10 possible comparisons, only five had relevant published data. The comparison PD versus HD hospital was however the comparison with most available data.

We found only one RCT [29] out of our 4,348 abstracts, which may be an indication that RCTs may not the be the most appropriate design for studying different types of dialysis. The reason for this could be both the strong wish from the patient for a specific treatment as well as the routines of the health professionals. While we were unable to include many RCTs in our SR, we have included 13 controlled observational studies (from 12 publications) with numbers of patients ranging from 28 to 11,238 .

We used GRADE to assess the quality of the evidence for each endpoint. In GRADE the observational studies start on low quality (due to lack of randomization). As soon as we had one additional reason to downgrade the quality fell to very low and this is reflected in the overall qualities of our endpoints.

We have only assessed studies with similar comorbidity (including diabetes mellitus) and age at baseline. Differences in comorbidity at baseline could have influenced the results of comparisons of different dialysis modalities. Based upon this and the fact that it has been shown to be very difficult to perform RCTs for this type of patients, we believe that our results from observational studies with low quality are, in practice, the best available we can obtain for this type of treatment.

In this study, we focused specifically on the type of dialysis given and the delivery location. Consequently, we could not examine differences in dialysis frequency, dialysis adequacy, residual function or dialysis equipment, all of which could have influenced our results. Even though we had no pre-de- 
fined focus on whether the patients were initial or established patients, five of the six observational studies we included for the comparison PD versus HD hospital were with initial patients and one was a mixture of incident and established dialysis patients. Further, sensitivity analyses showed no significant difference in mortality for studies with 1 year and 4 years observation period.

With regard to geographical conditions and existing infrastructure in different regions, it was difficult to obtain reliable cost information for the different modalities, particularly HD home and satellite. We have tried to conduct our analyses based on best available cost data, and have incorporated uncertainty around cost estimates in the sensitivity analysis. Further, we did not include the costs related to loss of production in our analysis as we assumed that most dialysis patients are of retirement age or out of work.

We also performed a systematic literature search to identify the best possible evidence on utilities for our model analysis. The search did not identify any single study or combination of studies reporting the utility values measured by a common instrument for all types of dialysis. Lacking good-quality utility data (preference-based health-related quality of life data) for our study population, we applied a single QALY weight for all types of dialysis, based on a non-randomized Swedish study, which attempted to control for case-mix between PD and HD patients [54]. However, because one could imagine that home or satellite dialysis patients might experience a higher quality of life than hospital HD patients, we examined the implication of a potentially higher quality of life associated with these modalities. The correction factor, however, had a very small effect on the results and the conclusion in terms of cost-effectiveness was unchanged.

\section{Conclusion and policy implications}

We found that over a 5-year time horizon, PD was the most cost-effective dialysis alternative and comparable with HD regarding the efficacy measured as mortality or complications. There are significant cost-saving potential if more patients start on PD instead of HD. Results from our SR and newly published literature indicate that starting more patients on PD can advantageously be done for the first 3 - 5 years of dialysis for patients at all ages without diabetes. However, patient's clinical status and preferences need to be considered in each individual case.

\section{Acknowledgments}

We thank research librarian Ingrid Harboe for her assistance in the literature searches and Arna Desser for her contribution.

\section{Financial Disclosure}

This work was supported by the Norwegian Knowledge Centre for the Health Services.

\section{Conflicts of Interest}

None of the authors have any conflicts of interest related to this manuscript.

\section{Author Contributions}

EP carried out the clinical part and drafted the manuscript. VH carried out the economical part and drafted the manuscript. TR participated in the clinical part and commented on the manuscript. TW performed the statistical analysis, co-developed the economic model and commented on the manuscript. MK was responsible for the project and participated in its design, coordination and drafting of the manuscript.

\section{References}

1. Andrassy KM. Comments on 'KDIGO 2012 Clinical Practice Guideline for the Evaluation and Management of Chronic Kidney Disease'. Kidney Int. 2013;84(3):622623.

2. Jha V, Garcia-Garcia G, Iseki K, Li Z, Naicker S, Plattner B, Saran R, et al. Chronic kidney disease: global dimension and perspectives. Lancet. 2013;382(9888):260-272.

3. Hallan SI, Coresh J, Astor BC, Asberg A, Powe NR, Romundstad S, Hallan HA, et al. International comparison of the relationship of chronic kidney disease prevalence and ESRD risk. J Am Soc Nephrol. 2006;17(8):22752284.

4. Tonelli M, Riella M. Chronic kidney disease and the aging population. Int J Organ Transplant Med. 2014;5(1):16.

5. Grassmann A, Gioberge S, Moeller S, Brown G. ESRD patients in 2004: global overview of patient numbers, treatment modalities and associated trends. Nephrol Dial Transplant. 2005;20(12):2587-2593.

6. Care FM. ESRD patients in 2012, a global perspective, 2012.

7. The Norwegian Renal Registry. Annual report 20122013 [Available from: www.nephro.no].

8. Liberati A, Altman DG, Tetzlaff J, Mulrow C, Gotzsche PC, Ioannidis JP, Clarke M, et al. The PRISMA statement for reporting systematic reviews and meta-analyses of studies that evaluate health care interventions: explanation and elaboration. J Clin Epidemiol. 2009;62(10):e134.

9. Higgins J, Green S. Cochrane Handbook for Systematic Reviews of Interventions. Cochrane Collaboration. 2011.

10. The GRADE working group [Available from: www. gradeworkinggroup.org].

11. The Norwegian Renal Registry. Annual reports 2013 [updated 2012. Available from: http:/www.nephro.no/nnr. html].

12. Nyhus k, Kristensen F, Mermejean P, Sverre J. Cost comparison between haemodialysis and peritoneal dialysis in Norway for patients who can use either treatment modal- 
ity. Value Health. 2007;10(6):A238.

13. Norwegian Medicines Agency. Legemiddelsok 2013 [Available from: http://www.legemiddelverket.no/ Legemiddelsoek/Sider/default.aspx].

14. Wisloff T, Selmer RM, Halvorsen S, Fretheim A, Norheim OF, Kristiansen IS. Choice of generic antihypertensive drugs for the primary prevention of cardiovascular disease - a cost-effectiveness analysis. BMC Cardiovasc Disord. 2012;12:26.

15. Bjorvatn A. Evaluering av desentralisert dialysebehandling, 2003.

16. Paulsen D, Solbakken K, Valset T. [Peritoneal dialysis at a regional hospital in Norway]. Tidsskr Nor Laegeforen. 2011;131(16):1547-1549.

17. NHO Service. Sammen for velferd i Time kommune [updated 2013. Available from: http://www.nho.no/getfile. php/Offentlig\%20sektor/Fylker/Rogaland/Regneeksempler/1121_Time.pdf].

18. Canaud B, Tong L, Tentori F, Akiba T, Karaboyas A, Gillespie B, Akizawa T, et al. Clinical practices and outcomes in elderly hemodialysis patients: results from the Dialysis Outcomes and Practice Patterns Study (DOPPS). Clin J Am Soc Nephrol. 2011;6(7):1651-1662.

19. Sennfalt K, Magnusson M, Carlsson P. Comparison of hemodialysis and peritoneal dialysis - a cost-utility analysis. Perit Dial Int. 2002;22(1):39-47.

20. Lunde L, Wisloff T. Do choice of outcome measure matter in cost-effectiveness analysis?(draft), 2011.

21. Korosec Jagodic H, Jagodic K, Podbregar M. Long-term outcome and quality of life of patients treated in surgical intensive care: a comparison between sepsis and trauma. Crit Care. 2006;10(5):R134.

22. Lonn for ansatte i private helse- og sosialtjenester, 2013 [Available from: http://www.ssb.no/lonnhelse/].

23. Norwegian Directorate of Health. Nasjonal faglig retningslinje for antibiotikabruk i sykehus 2013.

24. Baig SZ, Coats WC, Aggarwal KB, Alpert MA. Assessing cardiovascular disease in the dialysis patient. Adv Perit Dial. 2009;25:147-154.

25. Stack AG, Bloembergen WE. Prevalence and clinical correlates of coronary artery disease among new dialysis patients in the United States: a cross-sectional study. Jam Soc Nephrol. 2001;12(7):1516-1523.

26. Wisloff T, Abrahamsen TG, Bergsaker MA, Lovoll O, Moller P, Pedersen MK, Kristiansen IS. Cost effectiveness of adding 7-valent pneumococcal conjugate (PCV7) vaccine to the Norwegian childhood vaccination program. Vaccine. 2006;24(29-30):5690-5699.

27. Liem YS, Bosch JL, Hunink MG. Preference-based quality of life of patients on renal replacement therapy: a systematic review and meta-analysis. Value Health. 2008;11(4):733-741.

28. Wisloff T, Hagen G, Hamidi V, Movik E, Klemp M, Olsen JA. Estimating QALY gains in applied studies: a review of cost-utility analyses published in 2010. Pharmacoeconomics. 2014;32(4):367-375.

29. Korevaar JC, Feith GW, Dekker FW, van Manen JG, Boeschoten EW, Bossuyt PM, Krediet RT. Effect of starting with hemodialysis compared with peritoneal dialysis in patients new on dialysis treatment: a randomized controlled trial. Kidney Int. 2003;64(6):2222-2228.

30. Andrikos E, Tseke P, Balafa O, Pappas M. Five-year survival in comparable HD and PD patients: one center's experience. Int J Artif Organs. 2008;31(8):737-741.

31. Aslam N, Bernardini J, Fried L, Burr R, Piraino B. Comparison of infectious complications between incident hemodialysis and peritoneal dialysis patients. Clin J Am Soc Nephrol. 2006;1(6):1226-1233.

32. Ganeshadeva YM, Goh BL, Chew SE, Zahari MR. A single-center audit of complication rates between hemodialysis and peritoneal dialysis patients. Hemodialysis International. 2009; Conference(var.pagings):388-389.

33. Jager KJ, Merkus MP, Boeschoten EW, Dekker FW, Tijssen JG, Krediet RT. What happens to patients starting dialysis in the Netherlands? Neth J Med. 2001;58(4):163173.

34. Johansen KL, Zhang R, Huang Y, Chen SC, Blagg CR, Goldfarb-Rumyantzev AS, Hoy CD, et al. Survival and hospitalization among patients using nocturnal and short daily compared to conventional hemodialysis: a USRDS study. Kidney Int. 2009;76(9):984-990.

35. Kumar VA, Ledezma ML, Idroos ML, Burchette RJ, Rasgon SA. Hospitalization rates in daily home hemodialysis versus peritoneal dialysis patients in the United States. Am J Kidney Dis. 2008;52(4):737-744.

36. Lee EJ. Risk stratification of the peritoneal dialysis patient - a single-center experience. Perit Dial Int. 2008;28(Suppl 3):S32-35.

37. Roderick P, Nicholson T, Armitage A, Mehta R, Mullee M, Gerard K, Drey N, et al. An evaluation of the costs, effectiveness and quality of renal replacement therapy provision in renal satellite units in England and Wales. Health Technol Assess. 2005;9(24):1-178.

38. Verdalles U, Abad S, Aragoncillo I, Villaverde M, Jofre $\mathrm{R}$, Verde E, Vega A, et al. Factors predicting mortality in elderly patients on dialysis. Nephron Clin Pract. 2010;115(1):c28-34.

39. Vigneau C, Trolliet P, Labeeuw M, Pouteil-Noble C. [Which method of dialysis for the type 2 diabetic?]. Nephrologie. 2000;21(4):173-178.

40. Weinhandl ED, Liu J, Gilbertson DT, Arneson TJ, Collins AJ. Survival in daily home hemodialysis and matched thrice-weekly in-center hemodialysis patients. J Am Soc Nephrol. 2012;23(5):895-904.

41. Williams VR, Quinn R, Callery S, Kiss A, Oliver MJ. The impact of treatment modality on infection-related hospitalization rates in peritoneal dialysis and hemodialysis patients. Perit Dial Int. 2011;31(4):440-449.

42. Jain AK, Blake P, Cordy P, Garg AX. Global trends in rates of peritoneal dialysis. J Am Soc Nephrol. 2012;23(3):533544.

43. Heaf JG, Wehberg S. Relative survival of peritoneal dialysis and haemodialysis patients: effect of cohort and mode of dialysis initiation. PLoS One. 2014;9(3):e90119.

44. Marshall MR, Polkinghorne KR, Kerr PG, Hawley CM, Agar JW, McDonald SP. Intensive Hemodialysis and Mortality Risk in Australian and New Zealand Populations. Am J Kidney Dis. 2016;67(4):617-628. 
45. Marshall MR, Walker RC, Polkinghorne KR, Lynn KL. Survival on home dialysis in New Zealand. PLoS One. 2014;9(5):e96847.

46. Wu B, Wang M, Gan L, Zhao H. Comparison of patient survival between hemodialysis and peritoneal dialysis in a single Chinese center. Int Urol Nephrol. 2014;46(12):2403-2407.

47. Yang F, Khin LW, Lau T, Chua HR, Vathsala A, Lee E, Luo N. Hemodialysis versus Peritoneal Dialysis: A Comparison of Survival Outcomes in South-East Asian Patients with End-Stage Renal Disease. PLoS One. 2015;10(10):e0140195.

48. Filiopoulos V, Hadjiyannakos D, Papakostoula A, Takouli L, Biblaki D, Dounavis A, Vlassopoulos D. Long term outcome of hemodialysis versus peritoneal dialysis patients: 20-year experience from a single center. Nephrol Dial Transplant. 2014;29:iii520-iii21.

49. Couchoud C, Bolignano D, Nistor I, Jager KJ, Heaf J, Heimburger O, Van Biesen W. Dialysis modality choice in diabetic patients with end-stage kidney disease: a systematic review of the available evidence. Nephrol Dial Transplant. 2015;30(2):310-320.

50. Haller M, Gutjahr G, Kramar R, Harnoncourt F, Ober- bauer R. Cost-effectiveness analysis of renal replacement therapy in Austria. Nephrol Dial Transplant. 2011;26(9):2988-2995.

51. Howard K, Salkeld G, White S, McDonald S, Chadban S, Craig JC, Cass A. The cost-effectiveness of increasing kidney transplantation and home-based dialysis. Nephrology (Carlton). 2009;14(1):123-132.

52. Karopadi AN, Mason G, Rettore E, Ronco C. Cost of peritoneal dialysis and haemodialysis across the world. Nephrol Dial Transplant. 2013;28(10):2553-2569.

53. Villa G, Fernandez-Ortiz L, Cuervo J, Rebollo P, Selgas R, Gonzalez T, Arrieta J. Cost-effectiveness analysis of the Spanish renal replacement therapy program. Perit Dial Int. 2012;32(2):192-199.

54. Walker R, Marshall MR, Morton RL, McFarlane P, Howard $\mathrm{K}$. The cost-effectiveness of contemporary home haemodialysis modalities compared with facility haemodialysis: a systematic review of full economic evaluations. Nephrology (Carlton). 2014;19(8):459-470.

55. Treharne C, Liu FX, Arici M, Crowe L, Farooqui U. Peritoneal dialysis and in-centre haemodialysis: a cost-utility analysis from a UK payer perspective. Appl Health Econ Health Policy. 2014;12(4):409-420. 